\title{
GOLDIE CONDITIONS IN FINITE NORMALIZING EXTENSIONS
}

\author{
CHARLES LANSKI
}

\begin{abstract}
Let $S$ be a finite normalizing extension of a ring $R$. If $M$ is an $S$ module, then $M$ has finite uniform dimension if and only if it has finite uniform dimension when considered as an $R$ module. Consequently, when $S$ is a right Goldie ring, $R$ is also a right Goldie ring. Conversely, if $R$ is a semiprime right Goldie ring and $S$ is a prime ring, then $S$ is a Goldie ring. Finally, when both $S$ and $R$ are semiprime right Goldie rings, the quotient ring of $R$ embeds in the quotient ring of $S$.
\end{abstract}

In this paper we consider a finite normalizing extension $S$ of a ring $R$, and determine when the Goldie chain conditions transfer from one ring to the other. Results on chain conditions for this kind of extension have been obtained by Formanek and Jategaonkar [1], who showed that $S$ modules are Noetherian as $R$ modules exactly when they are Noetherian as $S$ modules; by Lemonnier [3], who proved a similar result for the Krull dimension of $S$ modules; and by Fisher, Lanski, and Park [2], who proved the corresponding theorem for Gabriel dimension. In the case that $S$ is a finite centralizing extension of $R$, Robson and Small [4] have obtained a number of results on chain conditions, as well as other properties of such extensions.

Throughout the paper, $S$ will denote a finite normalizing extension of a ring $R$. Thus $S=\sum_{i=1}^{n} s_{i} R$ with $s_{1}=1_{S}=1_{R}$ and $s_{i} R=R s_{i}$ for each $i$. All modules will be right unital modules. For an $R$ submodule $N$ of an $S$ module $M$, set $N^{t}=$ $\sum_{i=1}^{t} N s_{i}$.

Our first result is on finite uniform dimension and uses an idea of Lemonnier [3] to build $S$ modules from $R$ modules in $n$ successive steps. This theorem was obtained independently by J. C. Robson.

TheOREM 1. Any $S$ module $M$ has finite uniform dimension if and only if it has finite uniform dimension when considered as an $R$ module.

Proof. Since any $S$ submodule of $M$ is also an $R$ submodule, if $M_{R}$ has finite uniform dimension, then $M_{S}$ must have finite uniform dimension. For the other direction, we prove that any infinite direct sum of nonzero $R$ submodules of $M$ gives rise to an infinite direct sum of nonzero $S$ submodules of $M$.

Let $\left\{N_{i} \mid i=1,2, \ldots\right\}$ be a collection of nonzero $R$ submodules of $M$ so that $\Sigma N_{i}^{t}$ is a direct sum of the $R$ modules $\left\{N_{i}^{t}\right\}$, for some $t<n$. Consider first the possibility that $\left(\sum N_{i}^{t}\right) s_{t+1}^{-1} \cap \sum N_{i}=0$. In particular, $N_{i} s_{t+1} \neq 0$ for each $i$, and

Received by the editors August 31, 1979.

1980 Mathematics Subject Classification. Primary 16A34; Secondary 16A12. 
$\left(\sum N_{i} s_{t+1}\right) \cap \sum N_{i}^{t}=0$. If $\sum N_{i} s_{t+1}$ is a direct sum of $\left\{N_{i} s_{t+1}\right\}$, then $\sum N_{i}^{t+1}=$ $\Sigma\left(N_{i}^{t}+N_{i} s_{t+1}\right)$ is direct. Should $\Sigma N_{i} s_{t+1}$ not be direct, then for some $k$, and $n_{i} \in N_{i}$ one must have $n_{k+1} s_{t+1}=\sum_{i=1}^{k} n_{i} s_{t+1} \neq 0$. But then $n_{k+1}-n_{1}$ $-\cdots-n_{k} \in\left(\sum N_{i}^{t}\right) s_{t+1}^{-1} \cap \sum N_{i}=0$, contradicting the assumption that $\Sigma N_{i}^{t}$ is direct. Since we wish to produce an infinite collection of nonzero $R$ submodules $\left\{N_{i}\right\}$ so that $\Sigma N_{i}^{t+1}$ is direct, we may now assume that for any infinite subcollection $\left\{N_{j}\right\}$ of our original set $\left\{N_{i}\right\}$, that $\left(\Sigma N_{j}^{t}\right) s_{t+1}^{-1} \cap \Sigma N_{j} \neq 0$. Thus, for any $p>0$ there is $y \in N_{p+1} \oplus \cdots \oplus N_{k}, y \neq 0$, and $y s_{t+1} \in N_{p+1}^{t} \oplus \cdots \oplus N_{m}^{t}$. Let $q=$ $\max (k, m)$ and set $W=\left(N_{p+1} \oplus \cdots \oplus N_{q}\right) \cap\left(N_{p+1}^{t} \oplus \cdots \oplus N_{q}^{t}\right) s_{t+1}^{-1}$. Note that $W \neq 0$ since $y \in W$, and that $W^{t+1} \subset N_{p+1}^{t} \oplus \cdots \oplus N_{q}^{t}$. This observation enables one to produce an infinite collection of nonzero $R$ submodules $\left\{W_{i}\right\}$ of $M$ so that $\Sigma W_{i}^{t+1}$ is direct. Therefore, repetition of the argument above at most $n-t$ times gives an infinite set of nonzero $R$ submodules $\left\{M_{i}\right\}$ of $M$ so that $\Sigma M_{i}^{n}$ is direct. But $M_{i}^{n}=M_{i} S$ is an $S$ submodule of $M$, so the proof of the theorem is complete.

As an immediate consequence of Theorem 1, one has

THEOREM 2. If $S$ is a right Goldie ring, then $R$ is a right Goldie ring.

The converse to Theorem 2 is false, even when $R$ is a prime ring and $S$ is a semiprime ring. The following example shows that in such a situation $S$ need not satisfy either Goldie chain condition.

EXAMPLE 1. Let $R=F[X]$, the polynomial ring over a field $F$ in the infinite set of indeterminates $X=\left\{x_{1}, x_{2}, \ldots\right\}$. If $I$ is the ideal of $R$ generated by $\left\{x_{i} x_{j} \mid i \neq\right.$ $j\}$, set $S=R[y] / J$, for $J$ the ideal of $R[y]$ generated by $y^{2}-y$ and $y I$. Identifying $R$ with its image in $S$, one has

$$
S=R+R y=R+y F+\sum_{i} y x_{i} F\left[x_{i}\right]
$$

It is easy to see that $S$ is a semiprime ring, that $S y x_{i}=y x_{i} F\left[x_{i}\right]$, and that $\Sigma_{i} S y x_{i}$ is direct.

Even when $S$ is a semiprime Goldie ring and $R$ is a prime Goldie ring, there seems to be no reasonable relation between the uniform dimensions of $R$ and of $S$, and the number of generators of $S$ as an $R$ module. To demonstrate this, we present an easy example shown to us by $R$. Resco.

EXAMPLE 2. For $Z$ the ring of integers, let $S=Z \oplus(Z / m Z)$, and set $R=$ $Z(1, \overline{1}) \simeq Z$. Clearly, $S$ is generated over $R$ by two elements, the uniform dimension of $R$ is one, and if $m=p_{1} p_{2} \cdots p_{k}$ for distinct primes $p_{i}$, then the uniform dimension of $S$ is $k+1$.

For $S$ a prime Goldie ring one can obtain reasonable inequalities bounding the uniform dimension of $S$. These bounds will be consequences of our subsequent results.

In view of Example 1, our next theorem gives the best possible converse to Theorem 2. We wish to thank J. C. Robson for indicating how our proof for the prime case extended to the semiprime case. 
TheOREM 3. If $R$ is a semiprime right Goldie ring and $S$ is a prime ring, then $S$ is a right Goldie ring and $u-\operatorname{dim} S<n(u-\operatorname{dim} R)$, for $S=\sum_{i=1}^{n} s_{i} R$.

Proof. Let $R_{1}, \ldots, R_{t}$ be the distinct minimal annihilator ideals of $R$. If $R$ is a prime ring, then for consistency of notation, set $R_{1}=R$. For each $R_{i}$ choose $\left\{v_{i j}\right\} \subset\left\{s_{1}, \ldots, s_{n}\right\}$ maximal with respect to being right $R_{i}$ independent, so $F_{i}=\Sigma_{j} v_{i j} R_{i}$ is a free right $R_{i}$ module. The maximality of $\left\{v_{i j}\right\}$ means that for $s_{k} \notin\left\{v_{i j}\right\}, R_{i} \cap s_{k}^{-1} F_{i}=A_{i k} \neq 0$. For each such $s_{k}$ it is clear from the relation $s_{p} R=R s_{p}$ that $A_{i k}$ is an ideal of $R$, so the primeness of $R_{i}$ yields that $A_{i}=\cap_{k} A_{i k}$ is a nonzero ideal of $R$. Should $\left\{v_{i j}\right\}=\left\{s_{1}, \ldots, s_{n}\right\}$, set $A_{i}=R_{i}$. From the definition of $A_{i}, S A_{i}=\Sigma s_{j} A_{i} \subset F_{i}$. Since $R_{i} R_{j}=0$ for $i \neq j$, and since each $F_{i}$ is a torsion free $R_{i}$ module, $F=F_{1}+\cdots+F_{t}$ is a direct sum of $R$ modules and is a torsion free $R_{1} \oplus \cdots \oplus R_{t}$ module. In fact, $F$ is a torsion free $R$ module because if $c \in R$ is regular and $f c=0$ for $f \in F$, then $f c d=0$ for $d$ regular in $R_{1}$ $\oplus \cdots \oplus R_{t}$, and the regularity of $c d \in R_{1} \oplus \cdots \oplus R_{t}$ forces $f=0$. Also, note that if $A=A_{1} \oplus \cdots \oplus A_{t}$, then $S A \subset F$.

As each $A_{i}$ is a nonzero ideal of $R_{i}, A$ contains a regular element of $R$. Therefore $S / F$ is a torsion $R$ module, so it follows that if $Q$ is the semisimple Artinian right quotient ring of $R, F \otimes_{R} Q \cong S \otimes_{R} Q=M$ is a finitely generated right $Q$ module in which $F$ embeds as $F \otimes 1$. Letting $S$ act on $M$ by left multiplication gives a ring homomorphism of $S$ into $\operatorname{End}_{Q}(M)$. For any $y \in S, y \neq 0$, we have $y S A \neq 0$ because $S$ is a prime ring. But $y S A \subset S A \subset F$, which is a torsion free $R$ module. Therefore $y M=y S \otimes_{R} Q \supset y S A \otimes_{R} Q \neq 0$, and $S$ is isomorphic to a subring of $\operatorname{End}_{Q}(M)$. As a finitely generated $Q$ module, $M$ is a finite direct sum of irreducible $Q$ modules, so $\operatorname{End}_{Q}(M)$ is a semisimple Artinian ring. It follows that $S$ satisfies the ascending chain condition on right annihilators.

Let $\Sigma T_{i}$ be a direct sum of right ideals of $S$. For each $i, T_{i} S A$ is a nonzero right $R$ submodule of $F$, and $\Sigma_{i} T_{i} S A$ is direct. Now $F$ is a finite direct sum of $R$ submodules of the form $s_{j} R_{k}$, with $s_{j} R_{k} \cong R_{k}$ as $R_{k}$ modules. The uniform dimension of $R_{k}$ is the same when considered as either an $R$ module or as an $R_{k}$ module. Thus $u$-dim $\left(s_{j} R_{k}\right)_{R}=u$-dim $R_{k}$, so $F$ has finite uniform dimension, as an $R$ module, and in fact,

$$
u-\operatorname{dim} F_{R}<n \sum_{i} u-\operatorname{dim} R_{i}=n(u-\operatorname{dim} R) .
$$

Consequently $\left\{T_{i}\right\}$ contains at most $n(u$-dim $R)$ elements, so $S$ is a right Goldie ring.

In the situation of Theorem 3, or more generally when $R$ and $S$ are both semiprime right Goldie rings, it is natural to ask about the relation between the classical quotient rings of $R$ and of $S$. Our final theorem shows that $Q(R)$ does embed in $Q(S)$, although the embedding may not be unit to unit.

THEOREM 4. If $R$ and $S$ are both semiprime right Goldie rings, then (i) $Q(R)$ embeds in $Q(S)$, (ii) $u-\operatorname{dim} R<u-\operatorname{dim} S$, and (iii) if regular elements of $R$ are regular in $S$ then the embedding of $Q(R)$ in $Q(S)$ extends the natural embedding of $R$ in $Q(S)$. 
Proof. Let $T=\{q \in Q(S) \mid q c=0$ for $c$ a regular element of $R\}$, where we consider $R \subset S \subset Q(S)$. It is easy to see that $T$ is a $Q(S)-R$ sub-bimodule of $Q(S)$ and that $M=Q(S) / T$ is (right) $R$ torsion free. Note also that since $T$ is a left ideal of $Q(S)$, it is finitely generated, so $T d=0$ for some $d$ regular in $R$. Suppose that $M r=0$ for some $r \in R$. Then $Q(S) r \subset T$, so $Q(S) r d=0$, and $r=0$ follows from the regularity of $d$ in $R$. Therefore, $R$ embeds in $E=\operatorname{End}_{Q(s)}(M)$ via right multiplication on $M$. Furthermore, since $M$ is $R$ torsion free and $E$ is a semisimple Artinian ring, regular elements of $R$ are invertible in $E$, so $Q(R)$ embeds in $E$. Conclusion (i) follows from the fact that $M$ is isomorphic to a direct summand of $Q(S)$. The observation that $u$-dim $R=u$-dim $Q(R)$ is the cardinality of a set of primitive orthogonal idempotents proves (ii). Finally, if regular elements of $R$ are regular in $S$, then $T=0, E=Q(S)$, and (iii) is immediate.

We do not know under what general conditions the embedding of $Q(R)$ in $Q(S)$ will be unit to unit. It may be that this will hold whenever $S$ is a prime Goldie ring, since in this case it may be that regular elements of $R$ are regular in $S$. The following example based on a construction of G. Bergman, shows that in the semiprime case the embedding need not be unit to unit and that $Q(S)$ need not be generated as a module over the image of $Q(R)$.

EXAMPLE 3. Let $A$ be a commutative domain with 1 , and $g$ a homomorphism of $A$ onto itself so that $\operatorname{Ker}(g) \neq 0$. For example, take $A=F\left[x_{1}, x_{2}, \ldots\right]$ with $g$ generated by a downward shift of the indeterminates. Let $x \in \operatorname{Ker}(g)-\{0\}$ and set

$$
S=\left[\begin{array}{cc}
A & A \\
(x) & A
\end{array}\right] \oplus A
$$

For

$$
\bar{R}=\left[\begin{array}{cc}
A & A \\
(x) & A
\end{array}\right],
$$

let $R$ be the copy of $\bar{R}$ in $S$ under the isomorphism sending

$$
\left[\begin{array}{cc}
a & b \\
x c & d
\end{array}\right] \text { to }\left(\left[\begin{array}{cc}
a & b \\
x c & d
\end{array}\right], g(d)\right) \text {. }
$$

Then $S$ is a normalizing extension of $R$ generated by $1_{S}$ and $\left(0,1_{A}\right)$. Clearly, $Q(R) \cong M_{2}(K)$ for $K$ the ring of fractions of $A$, and $Q(S) \cong M_{2}(K) \oplus K$. Note that any embedding of $Q(R)$ in $Q(S)$ must send $Q(R)$ to $\left(M_{2}(K), 0\right)$, since otherwise the projection onto the second coordinate of $Q(S)$ would give an isomorphism of $M_{2}(K)$ onto $K$.

Using the inequality in Theorem 3 gives the following corollary of Theorem 4:

COROLlaRY. If $R$ is a semiprime right Goldie ring and $S$ is a prime ring, then $u-\operatorname{dim} R \leqslant u-\operatorname{dim} S \leqslant n(u-\operatorname{dim} R)$, for $S=\sum_{i=1}^{n} s_{i} R$.

We note that the lower bound in the corollary can be achieved by taking $S=M_{t}(F)$, for $F$ a field, and $R=F e_{11}+\cdots+F e_{t t}$, where $\left\{e_{i j}\right\}$ are the usual matrix units. Also, the upper bound can be attained, as the following example shows. 
ExAmple 4. Given $\boldsymbol{n} \geqslant 2$, let $\boldsymbol{F}$ be a field containing a primitive $\boldsymbol{n}$ th root of unity, say $w$. Let $S$ be the subring of $M_{n}(F[x])$ consisting of those matrices whose entries below the main diagonal belong to $x F[x]$. Clearly, $S$ is a prime Goldie ring with $u-\operatorname{dim} S=n$. If $\left\{e_{i j}\right\}$ are the usual matrix units in $M_{n}(F[x])$, let $T=e_{12}+e_{23}$ $+\cdots+e_{n-1 n}+x e_{n 1}$, and $Y=w e_{11}+w^{2} e_{22}+\cdots+w^{n} e_{n n}$. It follows that $R$, the $F$ subalgebra of $S$ generated by $I_{n}$ and $T$, is isomorphic to $F[x]$, that $w Y T=T Y$, and that $S=R+R Y+\cdots+R Y^{n-1}$ is a normalizing extension of $R$ with $u-\operatorname{dim} S=n(u-\operatorname{dim} R)$.

ADDED IN PROOF. The question about regular elements in $R$ being regular in $S$ has been shown to be false by an example of $\mathrm{L}$. Small. Using the notation of Example 3, let $R$ be the image of $A$ in $S=M_{2}(A)$ given by sending an $A$ to the diagonal matrix, $\operatorname{diag}(a, g(a))$. Then $S$ is a prime ring and a finite normalizing extension of $R$, but diag $(x, 0)$ is not regular in $S$.

\section{REFERENCES}

1. E. Formanek and A. Jategaonkar, Subrings of Noetherian rings, Proc. Amer. Math. Soc. 46 (1974), 181-186.

2. J. Fisher, C. Lanski and J. Park, Gabriel dimension of finite normalizing extensions, Comm. Algebra (to appear).

3. B. Lemonnier, Dimension de Krull et codeviation application au theoreme d'Eakin, Comm. Algebra G(16) (1978), 1647-1665.

4. J. C. Robson and L. Small, Liberal extensions, Proc. London Math. Soc. (to appear).

Department of Mathematics, University of Southern California, los Angeles, California 90007 